\title{
ON THE NUMBER OF REAL CURVES ASSOCIATED TO A COMPLEX ALGEBRAIC CURVE
}

\author{
EMILIO BUJALANCE, GRZEGORZ GROMADZKI, AND DAVID SINGERMAN
}

(Communicated by Clifford J. Earle, Jr.)

\begin{abstract}
Using non-Euclidean crystallographic groups we give a short proof of a theorem of Natanzon that a complex algebraic curve of genus $g \geq 2$ has at most $2(\sqrt{g}+1)$ real forms. We also describe the topological type of the real curves in the case when this bound is attained. This leads us to solve the following question: how many bordered Riemann surfaces can have a given compact Riemann surface of genus $g$ as complex double?
\end{abstract}

In [5] Natanzon proved, using topological methods, that a complex algebraic curve of genus $g \geq 2$ has at most $2(\sqrt{g}+1)$ real forms. He also showed that this bound is attained for infinitely many values of $g$, these being of the form $\left(2^{n-1}-1\right)^{2}$. Other formulations of this result are in terms of the associated compact Riemann surface $X$. An equivalent statement says that Aut $X$, the group of conformal and anticonformal automorphisms of $X$, has at most $2(\sqrt{g}+1)$ conjugacy classes of reflections, where by a reflection we mean an anticonformal involution with fixed points. Another formulation is that $X$ is the complex double of at most $2(\sqrt{g}+1)$ bordered Klein surfaces.

Here we use the combinatorial theory of non-Euclidean crystallographic (NEC) groups $[2,4]$ to give short proofs of these facts. We go further by showing that there are no other values of $g$ for which this bound is attained, and we describe the topological types of the real curves (or Klein surfaces) in question, showing that they have to be nonseparating (or nonorientable) with $2^{n-2}$ connected components. This leads us in Theorem 3 to solve a similar question about Riemann surfaces: how many bordered Riemann surfaces can have the same compact Riemann surface as their complex double?

It is well known $[1,2]$ that the categories of real algebraic curves and compact Klein surfaces are equivalent in the same way that the categories of complex algebraic curves and compact Riemann surfaces are equivalent. In the equivalence

Received by the editors July 31, 1991 and, in revised form, May 23, 1992.

1991 Mathematics Subject Classification. Primary 30F10; Secondary 14H55, $20 \mathrm{H} 10$.

The first author was partially supported by CICYT PB89-0201 and, with the third author, by Acciones Integradas (British/Spanish joint research programme).

(C) 1994 American Mathematical Society $0002-9939 / 94 \$ 1.00+\$ .25$ per page 
a real separating curve corresponds to an orientable bordered Klein surface, and a real nonseparating curve corresponds to a nonorientable bordered Klein surface. Also, complexification of real curves corresponds to complex doubles of Klein surfaces. Given these facts we can translate our theorems into results about real curves.

Theorem 1 (Natanzon). Let $Y_{1}, \ldots, Y_{k}$ be pairwise nonisomorphic compact bordered Klein surfaces of algebraic genus $g \geq 2$ with complex double $X$, and let $G=$ Aut $X$. Then

$$
\frac{2(g-1)}{|G|} \geq \frac{k-4}{4}
$$

and in particular $k \leq 2(\sqrt{g}+1)$.

Theorem 2. There exists a compact Riemann surface $X$ of genus $g \geq 2$ that is the complex double of $k=2(\sqrt{g}+1)$ nonisomorphic bordered Klein surfaces if and only if $g=\left(2^{n-1}-1\right)^{2}$ for some $n \geq 3$. Given such a Riemann surface $X$, Aut $X$ is the group generated by the $k$ reflections and is isomorphic to $\mathbb{Z}_{2}^{n+1}$. Furthermore, the $k$ Klein surfaces which have $X$ as complex double are all nonorientable with $2^{\text {n-2 }}$ boundary components.

In this section we prove Theorem 1. We use the algebraic structure of NEC groups. The basic results about these groups are explained in a recent book [2] which also includes references to the original papers. However, for the reader's convenience we point out a few of the concepts and properties that we use.

$$
\begin{aligned}
& \text { Every NEC group } \Lambda \text { has a signature } \\
& \left(g ; \pm ;\left[m_{1}, \ldots, m_{r}\right] ;\left\{C_{1}, \ldots, C_{k}\right\}\right) .
\end{aligned}
$$

For a group with this signature the quotient space $X=D / \Lambda$ (where $D$ is the hyperbolic plane) is a compact surface of genus $g$ with $k$ boundary components, and it is orientable if the + sign is used and nonorientable if the sign is used. The $m_{i}$ are integers $\geq 2$ called the periods, and they represent the branching over interior points of $X$. Each $C_{i}$, called the period cycles, is a cyclically ordered set of integers $\left(n_{i 1}, \ldots, n_{i s_{i}}\right)$ representing the branching over the $i$ th hole. The integers $n_{i j} \geq 2$ are called link periods. Associated with $C_{i}$, we have $s_{i}+1$ reflection generators $c_{i 0}, \ldots, c_{i s_{i}}$ and an orientation-preserving generator $e_{i}$ satisfying the relations

$$
\begin{aligned}
& c_{i 0}^{2}=c_{i 1}^{2}=\cdots=c_{i s_{i}}^{2}=\left(c_{i 0} c_{i 1}\right)^{n_{i 1}}=\cdots=\left(c_{i s_{i}-1} c_{i s_{i}}\right)^{n_{i s_{i}}}=1, \\
& c_{i s_{i}}=e_{i}^{-1} c_{i 0} e_{i} .
\end{aligned}
$$

As $c_{i s_{i}}$ is necessarily conjugate to $c_{i 0}$, we have at most $s_{i}$ conjugacy classes of reflections associated with this period cycle, and every reflection of $\Lambda$ is conjugate to one of the generating reflections. An empty period cycle has just one reflection generator associated with it, and reflection generators associated with two distinct period cycles cannot be conjugate. Every NEC group $\Lambda$ with the above signature has a fundamental region in $D$ whose hyperbolic area $\mu(\Lambda)$ is given by the formula

$$
\mu(\Lambda)=2 \pi\left(\varepsilon g-2+\sum_{i=1}^{r}\left(1-\frac{1}{m_{i}}\right)+k+\frac{1}{2} \sum_{i=1}^{k} \sum_{j=1}^{s_{i}}\left(1-\frac{1}{n_{i j}}\right)\right)
$$


where $\varepsilon=1$ if there is a - sign in the signature and $\varepsilon=2$ if there is a + sign.

If $\Gamma \subset \Lambda$ is a subgroup, then $|\Lambda: \Gamma|=\mu(\Gamma) / \mu(\Lambda)$. This is the area formula.

Proof of Theorem 1. The formula in Theorem 1 clearly holds if $k=1$, so let $k>1$, and represent $Y_{1}, \ldots, Y_{k}$ as orbit spaces $D / \Gamma_{1}, \ldots, D / \Gamma_{k}$, where $\Gamma_{i}$ are bordered surface NEC groups, i.e., NEC groups containing reflections and no other elements of finite order. Then $X=D / \Gamma$, where $\Gamma$ is a Fuchsian surface group and $\Gamma=\Gamma_{1}^{+}=\Gamma_{2}^{+}=\cdots=\Gamma_{k}^{+}$, where $\Gamma_{i}^{+}$is the canonical Fuchsian group of $\Gamma_{i}$, the subgroup of $\Gamma_{i}$ consisting of elements that preserve orientation. As $G=$ Aut $X$, we can write $G=\Lambda / \Gamma$ for some NEC group $\Lambda$. Thus $\Gamma<\Gamma_{i}<\Lambda$ for $i=1, \ldots, k$. We can choose for the coset representative of $\Gamma$ in $\Gamma_{i}$ a reflection $\gamma_{i}$, and as the $Y_{i}$ are pairwise nonisomorphic, the $\gamma_{i}$ are not conjugate in $\Lambda$. Also, each reflection of $\Lambda$ is conjugate to one of the generating reflections of $\Lambda$ associated to some period cycle as described above, so we can assume that $\gamma_{i}=c_{p q}$. Let $C_{1}, \ldots, C_{n}$ be all the different period cycles of $\Lambda$ involving these reflections, and assume that $C_{1}, \ldots, C_{t}$ are not empty and that $C_{t+1}, \cdots, C_{n}$ are empty. As each empty period cycle involves just one reflection, $C_{1}, \ldots, C_{t}$ involve $k-(n-t)$ reflections. Thus if $C_{i}=\left(n_{i 1}, \ldots, n_{i s}\right)$ then

$$
k-(n-t) \leq s_{1}+\cdots+s_{t},
$$

so from the formula (1) for the hyperbolic area $\mu(\Lambda)$ of a fundamental region for $\Lambda$ we obtain

$$
\mu(\Lambda) \geq 2 \pi(-2+n+(k-n+t) / 4) .
$$

Now $n \geq 1$, and if $t=0$, then all period cycles are empty and so $k=n$, and $k>1$. Otherwise $t \geq 1$, and in both cases we get

$$
\mu(\Lambda) \geq 2 \pi(k-4) / 4 .
$$

As $\Gamma$ is a Fuchsian surface group of genus $g$ that has index $|G|$ in $\Lambda$, we also have

$$
\mu(\Lambda)=\mu(\Gamma) /|G|=2 \pi(2 g-2) /|G|
$$

giving

$$
\frac{2(g-1)}{|G|} \geq \frac{k-4}{4},
$$

as claimed. As $|G| \geq 2 k$, we also obtain $k \leq 2(\sqrt{g}+1)$.

4

Before we prove Theorem 2, we review the method of determining the orientability of a subgroup of an NEC group generated by reflections. Let $\Lambda$ be an NEC group generated by reflections $c_{1}, \ldots, c_{s}$, and let $\Gamma_{0}<\Lambda$ be a subgroup of finite index. Form the Schreier coset graph $\mathscr{H}\left(\Lambda, \Gamma_{0}\right)$, whose vertices are the cosets $g \Gamma_{0}$ of $\Gamma_{0}$ in $\Lambda$ and where $g_{1} \Gamma_{0}$ is joined by an edge to $g_{2} \Gamma_{0}$ if and only if $c_{j} g_{1} \Gamma_{0}=g_{2} \Gamma_{0}$ for some generator $c_{j}$. Let $\overline{\mathscr{H}}\left(\Lambda, \Gamma_{0}\right)$ be the graph $\mathscr{H}\left(\Lambda, \Gamma_{0}\right)$ with the loops corresponding to reflection generators deleted. Then by Theorem 2(iii) of [3] we have 
Lemma. $\Gamma_{0}$ is nonorientable if and only if there is a closed path of odd length in $\overline{\mathscr{H}}\left(\Lambda, \Gamma_{0}\right)$.

We now prove Theorem 2 . We start by assuming the existence of a Riemann surface $X$ of genus $g$ admitting $k=2(\sqrt{g}+1)$ nonconjugate reflections. Let $G=$ Aut $X$ and $G_{0}$ be the subgroup generated by these reflections. Clearly $|G| \geq\left|G_{0}\right| \geq 2 k, k \geq 4$, and $g-1=k(k-4) / 4$. If $X=D / \Gamma$ and $G=\Lambda / \Gamma$ as above, then we have shown that $\mu(\Lambda) \geq 2 \pi(k-4) / 4$ so that $|G|=\mu(\Gamma) / \mu(\Lambda) \leq 2 k$, and hence $|G|=2 k$; in particular, $G=G_{0}$. Let $G=\left\{h_{1}, \ldots, h_{k}, g_{1}, \ldots, g_{k}\right\}$, where the $g_{i}$ are the reflections. Then $H=\left\{h_{1}, \ldots, h_{k}\right\}$ forms a subgroup so that, for $i=1, \ldots, k, h_{i} g_{1}$ is a reflection. Hence $\left(h_{i} g_{1}\right)^{2}=1$, or $h_{i} g_{1} h_{i}=g_{1}$. As the $g_{i}$ are nonconjugate, $h_{i} g_{1} h_{i}^{-1}=g_{1}$ so that $h_{i}^{2}=1$ for $i=1, \ldots, k$. Thus all the elements, besides 1 , of $G$ have order 2 so that $G$ is an abelian 2-group. Therefore, $G \cong \mathbb{Z}_{2}^{n+1}$, where $k=2^{n}$. As $k=2(\sqrt{g}+1), g=\left(2^{n-1}-1\right)^{2}$. We think of $G$ as $H \times \mathbb{Z}_{2}$, where $H$ is the subgroup of orientation-preserving automorphisms in $G$. Thus $G=\left\{\left(h_{j}, 1\right),\left(h_{j}, z\right)\right\}$, where $j=1, \ldots, k$ and $(1, z)$ is a reflection.

We now prove the existence of the surface $X$ of Theorem 2. (This proof is an algebraic analogue of Natanzon's proof in [5].) Let $\Lambda$ be a maximal NEC group of signature $(0 ;+;[] ;\{(2,2, \ldots, 2)\})$ with $k=2^{n}$ link periods equal to $2(n \geq 3)$. Such a group exists by [6], since the associated Fuchsian signature $(0 ;+;[2,2, \ldots, 2] ;\{\})$ is maximal. Also see [2, Theorem 2.4.7]. Notice that $\Lambda$ is just the group generated by reflections in the sides of a rightangled hyperbolic $k$-sided polygon so that $\Lambda$ is a right-angled Coxeter group. If $c_{1}, \ldots, c_{k}$ are the generating reflections, then $\Lambda$ has presentation

$$
\left\langle c_{1}, \ldots, c_{k} \mid c_{i}^{2}=1(i=1, \ldots, k),\left(c_{1} c_{2}\right)^{2}=\cdots=\left(c_{k-1} c_{k}\right)^{2}=\left(c_{k} c_{1}\right)^{2}=1\right\rangle .
$$

Define $\theta: \Lambda \rightarrow G$ by $\theta\left(c_{i}\right)=\left(h_{i}, z\right), i=1, \ldots, k$. Then $\Gamma=\operatorname{ker} \theta$ consists of orientation-preserving transformations and has no elements of finite order. Thus $\Gamma$ is a Fuchsian surface group, so $X=D / \Gamma$ is a Riemann surface admitting $k$ nonconjugate reflections, and, as above, the area formula shows that $k=2(\sqrt{g}+1)$, and then $g=\left(2^{n-1}-1\right)^{2}$.

We now have to show that, if $X$ is any Riemann surface of genus $g$ admitting $k=2(\sqrt{g}+1)$ nonconjugate reflections $g_{i}$, then the $k$ Klein surfaces $X /\left\langle g_{i}\right\rangle$ are nonorientable. (We can do this easily for the Riemann surface just constructed, for we let $\Gamma_{i}=\theta^{-1}\left\langle h_{i}, z\right\rangle$. Then $\Gamma<\Gamma_{i}<\Lambda$ with $\left|\Gamma_{i}: \Gamma\right|=2$. As $\prod_{i=1}^{k} h_{i}=1, c_{1} c_{2} \cdots c_{i-1} c_{i+1} \cdots c_{k} \in \Gamma_{i}$, and, as $k$ is even, the lemma implies that $\Gamma_{i}$ is nonorientable.) Now let $X$ be any Riemann surface of genus $g$ admitting $k=2(\sqrt{g}+1)$ nonconjugate reflections. As we saw, $X=D / \Gamma$ admits a group $G \cong Z_{2}^{n+1}$ of automorphisms, and hence there exists an epimorphism $\phi: \Lambda \rightarrow Z_{2}^{n+1}$ with kernel $\Gamma$, where $\Lambda$ is some NEC group. Thus $\Lambda$ can only have periods and link periods equal to 2 and so has signature of the form $\left(g ; \pm ;\left[2^{(r)}\right] ;\left\{\left(2^{\left(s_{1}\right)}\right), \ldots,\left(2^{\left(s_{t}\right)}\right),()^{v}\right\}\right)$. As $\Lambda$ has at leas̀t $k$ conjugacy classes of reflections, $v+\sum_{i=1}^{t} s_{i} \geq k$, and from above, $\mu(\Lambda)=2 \pi(k-4) / 4$. Using the area formula (1), we see that $t=1$, and then $\Lambda$ must have signature $\left(0 ;+;[] ;\left\{\left(2^{(k)}\right)\right\}\right)$. With the above presentation, the $k$ reflections of $X$ are $\phi\left(c_{i}\right) \quad(i=1, \ldots, k)$. Let $g_{u}=\phi\left(c_{u}\right)$ be one of these. Regard $\mathbb{Z}_{2}^{n+1}$ as a vector space of dimension $n+1$ over $\mathbb{Z}_{2}$, and choose a basis $g_{i_{0}}=\phi\left(c_{i_{0}}\right) \cdots g_{i_{n}}=\phi\left(c_{i_{n}}\right)$ 
not including $g_{u}$. Then $g_{u}$ is a linear combination of the $g_{i_{j}}$, say

$$
g_{u}=g_{i_{1}}+\cdots+g_{i_{t}} \quad \text { with } t>1
$$

As $c_{i_{1}} c_{i_{2}} \cdots c_{i_{t}} c_{u} \in \Gamma$ and $\Gamma$ is a Fuchsian surface group, $t$ is odd. Let $\Gamma_{u}=$ $\phi^{-1}\left(\left\langle g_{u}\right\rangle\right)$, and consider the Schreier coset graph $\mathscr{H}\left(\Lambda, \Gamma_{u}\right)$. As $g_{u} \neq g_{i_{k}}$ $(1 \leq k \leq t)$, the closed path corresponding to $c_{i_{1}} \cdots c_{i_{t}} c_{u}$ has no loops, for a loop would mean that $h^{-1} c_{i_{k}} h \in \Gamma_{u}(h \in \Lambda)$, and as $\Gamma_{u} \triangleleft \Lambda, c_{i_{k}} \in \Gamma_{u}$, which is not the case. Hence by the lemma, $\Gamma_{u}$ is nonorientable. As all Klein surfaces whose complex double is $X$ have this form, they must necessarily be nonorientable. Finally, by Theorem 2.3.3 and Remark 2.3.7 of [2] we see that all these surfaces have $2^{n-2}$ boundary components.

In Theorems 1 and 2 we solved the problem of finding the maximum number of bordered Klein surfaces whose complex double is a given Riemann surface $X$ of genus $g$. There is, of course, a more classical question involving bordered Riemann surfaces (or, equivalently, bordered orientable Klein surfaces).

Theorem 3. (i) Let $Y_{1}, \ldots, Y_{k}$ be nonisomorphic compact bordered Riemann surfaces with complex double $X$ of genus $g$. Then $(k-4) \cdot 2^{k-3} \leq g-1$, and this bound is attained for any pair $(g, k)$ satisfying $(k-4) \cdot 2^{k-3}=g-1$ with $k>4$.

(ii) If $X$ is a surface for which this bound is attained, then Aut $X$ is the group generated by the $k$ reflections of $X$ and is isomorphic to $\mathbb{Z}_{2}^{k}$, and also each surface $Y_{i}$ has $2^{k-3}$ boundary components.

Proof. (i) Let $Y_{i}=D / \Gamma_{i}$ for some bordered NEC group $\Gamma_{i}$ with $\Gamma_{i}^{+}=\Gamma$, where $\Gamma$ is a Fuchsian surface group such that $X=D / \Gamma$. Let $g_{i}=\gamma_{i} \Gamma \in \Gamma_{i} / \Gamma$ with $\gamma_{i}$ a reflection. If $G=$ Aut $X$, then $G=\Lambda / \Gamma$, where $\Lambda$ is an NEC group. As in the proof of Theorem 1, we may assume that $\gamma_{1}, \ldots, \gamma_{k}$ are conjugate canonical reflections $c_{1}, \ldots, c_{k}$ of $\Lambda$ and that $\mu(\Lambda) \geq 2 \pi(k-4) / 4$.

We now show that $|G| \geq 2^{k}$ by proving that the set of elements of $\Lambda / \Gamma$ of the form

$$
c_{i_{1}} c_{i_{2}} \cdots c_{i_{r}} \Gamma \quad\left(1 \leq i_{1}<i_{2}<\cdots<i_{r} \leq k\right)
$$

are distinct. Suppose that

$$
c_{i_{1}} c_{i_{2}} \cdots c_{1_{r}} \Gamma=c_{j_{1}} c_{j_{2}} \cdots c_{j_{s}} \Gamma
$$

where $i_{1}<i_{2}<\cdots<i_{r}, j_{1}<j_{2}<\cdots<j_{s}$, and $\left\{i_{1}, \ldots, i_{r}\right\} \neq\left\{j_{1}, \ldots, j_{2}\right\}$. As each $c_{k_{e}}$ normalizes $\Gamma$, we may assume that $i_{r} \neq j_{s}$ and without loss of generality that $i_{r}=\max \left(i_{r}, j_{s}\right)$. Then $i_{r} \neq j_{u} \quad(1 \leq u \leq s)$. Now

$$
c_{i_{r}} c_{i_{r-1}} \cdots c_{i_{1}} c_{j_{1}} \cdots c_{j_{s}} \in \Gamma
$$

and as $\Gamma$ is a Fuchsian group, $r+s$ is even. Also $c_{i_{r-1}} \cdots c_{i_{1}} c_{j_{1}} \cdots c_{j_{s}} \in \Gamma_{i_{r}}$, and this word corresponds to a closed path of odd length in the Schreier coset graph $\mathscr{H}\left(\Lambda, \Gamma_{i_{r}}\right)$. We show that this path has no loops. There is a loop at $c_{w}\left(w \in\left\{i_{u}, j_{v} \mid u<r, v \leq s\right\}\right)$ if $c_{w}$ fixes a coset $h \Gamma_{i_{r}}$. If this occurs, then $h^{-1} c_{w} h \in \Gamma_{i_{r}}$ and so

$$
\Gamma_{i_{r}}=\Gamma \cup h^{-1} c_{w} h \Gamma=h^{-1}\left(\Gamma \cup c_{w} \Gamma\right) h=h^{-1} \Gamma_{w} h
$$


This is impossible, as $Y_{w}$ and $Y_{i_{r}}$ are not isomorphic. Thus the path has no loops, and by the lemma in $\S 4, \Gamma_{i_{r}}$ is nonorientable, a contradiction. Thus $|G| \geq 2^{k}$, and as

$(*) \quad \frac{2 \pi(2 g-2)}{|G|}=\frac{\mu(\Gamma)}{|G|}=\mu(\Lambda) \geq \frac{2 \pi(k-4)}{4}, \quad(k-4) \cdot 2^{k-3} \leq g-1$.

Now let $(g, k)$ be a pair of positive integers satisfying the above inequality with $k>4$. Let $\Lambda$ be a maximal NEC group of signature $(0 ;+;[]$; $\{(2,2, \ldots, 2)\})$ with $k$ link periods equal to 2 and with generating reflections $c_{1}, \ldots, c_{k}$ (as in $\S 4$ ). Define a homomorphism $\theta: \Lambda \rightarrow \mathbb{Z}_{2}^{k}$ by $\theta\left(c_{i}\right)=g_{i}$, where $\left\{g_{1}, \ldots, g_{k}\right\}$ is a basis for $\mathbb{Z}_{2}^{k}$. Then $\operatorname{ker} \theta=\Gamma$ is a Fuchsian group and $X=D / \Gamma$ is a Riemann surface admitting $k$ reflections. The area formula gives $(k-4) \cdot 2^{k-3}=g-1$. Let $\Gamma_{i}=\theta^{-1}\left(\left\langle g_{i}\right\rangle\right)$ so that $\Gamma_{i} \triangleleft \Lambda$. We show that $\Gamma_{i}$ is orientable. As in the proof of Theorem 2, a loop in a path in $\mathscr{H}\left(\Lambda, \Gamma_{i}\right)$ corresponds to $c_{i}$, being part of the corresponding word. Thus a closed path of odd length in $\overline{\mathscr{H}}\left(\Lambda, \Gamma_{i}\right)$ corresponds to a word $c_{i_{1}} c_{i_{2}} \cdots c_{i_{r}} \in \Gamma_{i}$ with $i_{u} \neq i \quad(1 \leq u \leq r)$ and $r$ odd. We can then find a linear dependence relation among $g_{1}, \ldots, g_{k}$, which is a contradiction. Thus the $k$ surfaces $D / \Gamma_{i}$ are all orientable.

(ii) Now let $X$ be a compact Riemann surface of genus $g>1$ admitting $k$ nonconjugate reflections $g_{1}, \ldots, g_{k}$ such that $X /\left\langle g_{i}\right\rangle$ are orientable, and furthermore suppose that $(k-4) \cdot 2^{k-3}=g-1$. As before, we let $X=D / \Gamma$, where $\Gamma$ is a Fuchsian surface group and find $k$ reflections $c_{1}, \ldots, c_{k}$ which are lifts of $g_{1}, \ldots, g_{k}$ and thus normalize $\Gamma$. As we have seen previously, $c_{i_{1}} c_{i_{2}} \cdots c_{i_{r}} \Gamma$ are distinct cosets $\left(1 \leq i_{1}<i_{2}<\cdots<i_{r} \leq k\right)$, so $|G| \geq 2^{k}$, where $G=$ Aut $X$. However, if we use the above relation between $g$ and $k$ in $(*)$, we find that $|G| \leq 2^{k}$, and so $|G|=2^{k}$. We now show that $g_{1}, \ldots, g_{k}$ commute with each other. Let $i<j$, and consider $g_{j} g_{i}=c_{j} c_{i} \Gamma$. There exist $u_{1}, \ldots, u_{t}\left(1 \leq u_{1}<u_{2}<\cdots<u_{t} \leq k\right)$ such that

$$
c_{j} c_{i} \Gamma=c_{u_{1}} c_{u_{2}} \cdots c_{u_{t}} \Gamma
$$

and hence

$$
c_{i} c_{j} c_{u_{1}} \cdots c_{u_{t}} \in \Gamma
$$

thus, $t$ is even. Now suppose that, for some $a(1 \leq a \leq t), c_{u_{a}} \neq c_{i}$ and $c_{u_{a}} \neq c_{j}$. Then as each $c_{j}$ normalizes $\Gamma$,

$$
c_{i} c_{j} \cdots c_{u_{a-1}} c_{u_{a+1}} \cdots c_{u_{t}} \in \Gamma_{u_{a}}
$$

As in the proof of part (i), this shows that there is a closed path of odd length in $\overline{\mathscr{H}}\left(\Lambda, \Gamma_{u_{a}}\right)$, which is impossible, as $\Gamma_{u_{a}}$ is orientable. Hence, $g_{j} g_{i}=$ $c_{j} c_{i} \Gamma=c_{i} c_{j} \Gamma=g_{i} g_{j}$ and thus $G \cong \mathbb{Z}_{2}^{k}$. As $|G|=2^{k}, G \cong \Lambda / \Gamma$, where $\mu(\Lambda)=2 \pi(k-4) / 4$, and as in the proof of Theorem $2, \Lambda$ has signature $(0 ;+;[] ;\{(2, \ldots, 2)\})$ with $k$ link periods equal to 2 . The homomorphism from $\Lambda$ to $\mathbb{Z}_{2}^{k}$ with kernel $\Gamma$ is unique up to choosing the basis of $\mathbb{Z}_{2}^{k}$, and as in Theorem 2 we show that each $X /\left\langle g_{i}\right\rangle$ has $2^{n-3}$ boundary components.

\section{ACKNOWLEDGMENT}

We thank the referee, Clifford Earle, for some helpful comments on an earlier version of this paper. 


\section{REFERENCES}

1. N. L Alling and N. Greenleaf, Foundations of the theory of Klein surfaces, Lecture Notes in Math., vol. 219, Springer-Verlag, New York, 1971.

2. E. Bujalance, J. J. Etayo, J. M. Gamboa, and G. Gromadzki, Automorphism groups of compact bordered Klein surfaces, Lecture Notes in Math., vol. 1439, Springer-Verlag, New York, 1990.

3. A. H. M. Hoare and D. Singerman, The orientability of subgroups of plane groups, Groups (St. Andrews, 1981), London Math. Soc. Lecture Notes Ser., vol. 71, Cambridge Univ. Press, Cambridge and New York, 1982, pp. 221-227.

4. A. M. Macbeath, The classification of non-Euclidean plane crystallographic groups, Canad. J. Math. 19 (1967), 1192-1205.

5. S. M. Natanzon, On the order of a finite group of homeomorphisms of a surface onto itself and the number of real forms of a complex algebraic curve, Soviet Math. Dokl. 19 (1978), 1195-1199.

6. D. Singerman, Finitely maximal Fuchsian groups, J. London Math. Soc. (2) 6 (1972), 29-38.

Facultad de Ciencias, Departamento de Matemáticas Fundamentales, Universidad Nacional de Educación a Distancia, 28040 Madrid, Spain

E-mail address: Emilio.Bujalance@Human.uned.es

Institut Matematyki WSP, Chodkiewicza 30, 85-064 Bydgoszcz, Poland

Faculty of Mathematical Studies, University of Southampton, S09 5NH SouthampTON, ENGLAND

E-mail address: ds@uk.ac.soton.maths 